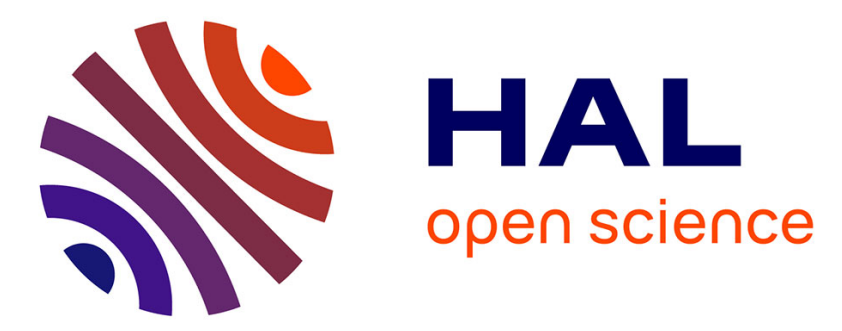

\title{
Using $\gamma$ rays to disentangle fusion-fission and quasifission near the Coulomb barrier: A test of principle in the fusion-fission and quasielastic channels
}

E. Vardaci, A. Pulcini, E.M. Kozulin, I. Matea, D. Verney, A. Maj, C. Schmitt, I.M. Itkis, G.N. Knyazheva, K. Novikov, et al.

\section{To cite this version:}

E. Vardaci, A. Pulcini, E.M. Kozulin, I. Matea, D. Verney, et al.. Using $\gamma$ rays to disentangle fusion-fission and quasifission near the Coulomb barrier: A test of principle in the fusion-fission and quasielastic channels. Phys.Rev.C, 2020, 101 (6), pp.064612. 10.1103/PhysRevC.101.064612 . hal02892938

\section{HAL Id: hal-02892938 \\ https://hal.science/hal-02892938}

Submitted on 1 Dec 2020

HAL is a multi-disciplinary open access archive for the deposit and dissemination of scientific research documents, whether they are published or not. The documents may come from teaching and research institutions in France or abroad, or from public or private research centers.
L'archive ouverte pluridisciplinaire HAL, est destinée au dépôt et à la diffusion de documents scientifiques de niveau recherche, publiés ou non, émanant des établissements d'enseignement et de recherche français ou étrangers, des laboratoires publics ou privés. 


\title{
Using $\gamma$-rays to disentangle Fusion-Fission and Quasi-Fission near the Coulomb barrier: a test of principle in the Fusion-Fission and Quasi-Elastic channels
}

\author{
E. Vardaci,,${ }^{1,2, *}$ A. Pulcini,,${ }^{1,2}$ E. M. Kozulin, ${ }^{3}$ I. Matea,${ }^{4}$ D. Verney, ${ }^{4}$ A. Maj, ${ }^{5}$ C. Schmitt,${ }^{6}$ I.M. Itkis,${ }^{3}$ \\ G.N. Knyazheva, ${ }^{3}$ K. Novikov ${ }^{3}$ N. Kozulina, ${ }^{3}$ I.M. Harca, ${ }^{3}$ I.V. Kolesov, ${ }^{3}$ K. Saveleva, ${ }^{3}$ V.V. Kirakosyan, ${ }^{3}$ \\ O. Dorvaux,${ }^{7}$ M. Ciemala,${ }^{5}$ S. Brambilla, ${ }^{8}$ M. Ashaduzzaman, $,{ }^{1,2}, 9$ B. De Canditiis,,${ }^{1,2}$ A. Di Nitto,,${ }^{1,2}$ \\ D. Quero, ${ }^{1,2}$ C. Parascandolo, ${ }^{2}$ D. Pierroutsakou, ${ }^{2}$ P.K. Rath,${ }^{1,2,10}$ G. Sposito, ${ }^{1,2}$ G. La Rana, ${ }^{1,2}$ \\ A. Bracco, ${ }^{8,11}$ F. Camera, ${ }^{8,11}$ O. Stezowski, ${ }^{8,11}$ C. Borcea,${ }^{12}$ S. Calinescu, ${ }^{12}$ C. Petrone, ${ }^{12}$ and J. Wilson ${ }^{4}$ \\ ${ }^{1}$ Dipartimento di Fisica "E. Pancini", Universitá degli Studi di Napoli "Federico II", 80126 Napoli, Italy \\ ${ }^{2}$ Istituto Nazionale di Fisica Nucleare, Sezione di Napoli, 80126 Napoli, Italy \\ ${ }^{3}$ Flerov Laboratory of Nuclear Reaction, Joint Institute for Nuclear Research, 141980 Dubna, Moscow region, Russia \\ ${ }^{4}$ IPN, CNRS/IN2P3, Universitè Paris-Saclay, 91405 Orsay, France \\ ${ }^{5}$ Institute of Nuclear Physics PAN, Krakow, Poland \\ ${ }^{6}$ IPHC, 67037 Strasbourg, France \\ ${ }^{7}$ IPHC, CNRS/IN2P3, Universitè de Strasbourg, 67037 Strasbourg, France \\ ${ }^{8}$ Istituto Nazionale di Fisica Nucleare, Sezione di Milano, Milano, Italy \\ ${ }^{9}$ Department of Electrical and Electronic Engineering, \\ Green University of Bangladesh, Dhaka, Bangladesh \\ ${ }^{10}$ Centurion University, Paralakhemundi, 761211 Odisha, India \\ ${ }^{11}$ Università degli Studi di Milano, Milano, Italy \\ ${ }^{12}$ Horia Hulubei National Institute for $R \& \mathcal{E}$ in Physics and Nuclear Engineering (IFIN-HH), Bucharest - Magurele, Romania
}

\begin{abstract}
The overlap in the mass symmetric region of the reaction products from fusion-fission and quasifission complicates the assignment of symmetric events to complete fusion on the basis of the mass distribution alone. Additional observables, besides mass distribution, should be used. The approach proposed here relies on the fact that fusion-fission and quasi-fission are characterized by a different timescale. Within this framework, we performed a detailed study to find out how timescales can be probed via angular momentum transfer as measured via $\gamma$-ray multiplicities. The proof of principle was carried out by measuring the $\gamma$-rays in coincidence with fusion-fission and quasi-elastic binary fragments in the reaction ${ }^{32} \mathrm{~S}+{ }^{197} \mathrm{Au}$ at beam energy near the Coulomb barrier. The experiment was performed at the Tandem ALTO accelerator facility at IPN Orsay (France) using a detection setup consisting of ORGAM and PARIS $\gamma$-detector arrays coupled with the CORSET time-of-flight spectrometer. Results on the sensitivity of this method to distinguish reaction channels with different dynamics are discussed.
\end{abstract}

PACS numbers: $24.75 .+\mathrm{i} ; 25.70 . J \mathrm{j}$

\section{INTRODUCTION}

Heavy-ion induced fusion reactions at bombarding energies around the Coulomb barrier have been very successfully used for the production of superheavy elements (SHE) [1-6]. To select the optimal reactions for the production of new elements heavier than $\mathrm{Og}$ and/or new isotopes around the superheavy island of stability the measurement of fusion cross sections is an essential step [7]. In fusion events, a compound nucleus (CN) may evolve toward fission (fusion-fission, $\mathrm{FF}$ ) or become an evaporation residue (fusion-evaporation, ER) after the evaporation of light particles. The fusion cross section is measured by summing the cross sections of these two decay channels. In reactions involving medium mass nuclei the fission and evaporation cross sections can be of about the same magnitude $[8,9]$. This particular condition constitutes an advantage in the study of fission dynamics of medium mass nuclei $[10,11]$.

\footnotetext{
* corresponding author e-mail: vardaci@na.infn.it
}

In the case of reactions between massive nuclei necessary for the search of superheavy elements, the evaporation residues cross section is negligible with respect to the fission one. Therefore, it is sufficient to select and count the fission events to estimate the fusion cross section [12]. Fission events are selected by detecting in coincidence fragments produced in binary reactions. However, at energies around the Coulomb barrier, the quasifission $(\mathrm{QF})$ reaction mechanism, which also gives rise to binary products, becomes dominant and counteracts the complete fusion [12]. Furthermore, the mass distribution of binary products of QF reactions, due to the high production yield, can dominate the fragments symmetric mass region where also FF events are expected. Consequently, in the case of reactions between massive nuclei, the estimate of fusion cross sections, based on counting symmetric or nearly symmetric mass split events, can be biased because of the overlap of QF and FF binary events. Even if in the asymmetric region of the mass distribution it is possible to disentangle, to some extent, the components of QF and asymmetric FF modes, this is not possible in the symmetric mass region where the two 
processes are overlapped. Therefore, the overlap of QF and $\mathrm{FF}$ events constitutes an inescapable problem when CN cross sections have to be estimated [13].

Within the present work, we investigate if additional observables can be employed to unambiguously separate the FF and QF products, at least to some extent. In our view, these additional observables should reflect the differences between the slower dynamical evolution of the system passing through an equilibrium stage giving rise to the FF products, and the faster QF mechanism where the mass transfer and energy dissipation are strongly driven by shell effects [13-15]. Many experimental and theoretical works aimed at estimating the timescale of $\mathrm{FF}$ and QF confirm that QF takes place on a time scale of $\lesssim 10^{-20}$ s, whereas FF typically occurs on longer time scales, from $\sim 10^{-19}$ s to $\sim 10^{-16}$ s $[16-23]$.

It has been demonstrated by series of works [24-31], that pre-scission light particles can provide the timescale of the dynamical evolution of the compound nucleus from its formation up to the scission in two fragments. However, their emission probability is negligible in the typical reactions used for the SHE production in the FF and QF channels. A possible difference among the QF and FF paths can be found in the angular momentum dissipated during the dynamical evolution of the composite intermediate system, up to the reseparation stage, where the fragments production occurs. The entrance channel orbital angular momentum is fully transferred into internal degrees of freedom of the compound nucleus in FF, whereas only a fraction of it is available for the QF products, being the QF process faster [32-34]. It is reasonable to suspect that the fragments of $F F$ may reach a higher spin than those produced by QF. Consequently, the $\gamma$ ray multiplicity distributions $M_{\gamma}$, namely, the distribution of the number of $\gamma$-rays emitted per event, should reach higher average values in the case of $\mathrm{FF}$ events.

Independent information about low and high angular momentum transfer paths (respectively, QF and FF channels) can be extracted from discrete $\gamma$ transitions as well as from the $\gamma$-rays multiplicity in binary events. Hence, by measuring the $\gamma$-rays in coincidence with binary fragments a disentanglement of $\mathrm{QF}$ and $\mathrm{FF}$ might be attained. Therefore, the observable we focus on here are the $\gamma$-rays and their multiplicity $M_{\gamma}$ in the binary channels.

In order to test this method, namely the separation of a faster process from a slower one by $\gamma$-ray multiplicity, the reaction ${ }^{32} \mathrm{~S}+{ }^{197} \mathrm{Au}$, at the bombarding energy $\mathrm{E}_{L A B}=$ $166 \mathrm{MeV}$, near the Coulomb barrier $\mathrm{V}_{\text {Coul }}\left(\mathrm{E}_{C M} / \mathrm{V}_{\text {Coul }}\right.$ $=1.01$ ), was carried out at the Tandem ALTO accelerator facility at IPN Orsay (France). This reaction is characterized by a large FF cross section and a negligible QF cross section [35, 36]. At the same time, this reaction has a dominant contribution from the quasi-elastic (QE) channel which is governed by transfers of few nucleons and a relatively small energy and angular momentum dissipation [37, 38]. The FF and QE channels can be well separated by exploiting the large differences in the mass and total kinetic energy (TKE) distributions. Binary products of $\mathrm{QE}$ reactions are located in the mass region around the mass of the target and projectile nuclei and their TKE is about equal to the entrance channel relative motion energy. In FF, the mass distribution is centered around symmetric splitting and has a characteristic width [39]. The TKE distribution is instead expected to follow Viola systematics [40]. Because of these important differences, this proposed reaction represents an suitable benchmark to characterize the $\gamma$-rays multiplicities dependence on reaction time-scales by using the measurements of $\gamma$-rays in coincidence with the binary fragments assigned to the $\mathrm{QE}$ and $\mathrm{FF}$, i.e. from the faster and slower processes, respectively.

If the hypothesis that a higher angular momentum population corresponds to a larger interaction time is valid, the $\gamma$ transitions measured in coincidence with the QE component should come from nuclei populated to lower angular momentum regions and the $\gamma$ multiplicity should be smaller than the one in coincidence with the fragments in the symmetric mass region. This would prove the concept and would open the road to experiments to distinguish between QF and FF in the symmetric region by employing an additional probe.

After a brief description of the experimental setup, the result of this approach will be discussed.

\section{EXPERIMENTAL SETUP}

A $350 \mu \mathrm{g} / \mathrm{cm}^{2}$ thick self-supporting ${ }^{197} \mathrm{Au}$ target was bombarded with a ${ }^{32} \mathrm{~S}$ beam at $E_{L A B}=166 \mathrm{MeV}$. The center of mass energy $E_{C M}$ is such that $\mathrm{E}_{C M} / \mathrm{V}_{\text {Coul }}=$ 1.01. The Tandem accelerator provided, on average, a continuous beam of intensity of $70 \mathrm{nA}$ during the 2 days long measurement.

Fragments from binary reactions were detected in coincidence by the two-arms TOF spectrometer CORSET [41]. Each arm consists of a micro channel plate (MCP) and a position-sensitive MCP acting as start and stop detectors, respectively. The arms were placed at $68^{\circ}$ and $-66.5^{\circ}$ with respect to the beam axis to maximize symmetric fragments detection. Each start detector covers an area of $20 \times 30 \mathrm{~mm}^{2}$ and was placed at $6 \mathrm{~cm}$ from the target. Each position sensitive stop detector covers an area of $60 \times 40 \mathrm{~mm}^{2}$ and was mounted $21 \mathrm{~cm}$ away from the start detector. In the event-by-event off-line analysis the position and time-of-flight of the fragments are translated into fragment mass numbers $A_{1,2}$, velocity vectors $\overrightarrow{v_{1}}, \overrightarrow{v_{2}}$ and TKE by employing standard two-body conservation laws [41]. With a time-of-flight resolution of $150 \mathrm{ps}$ and an angular resolution of $0.3^{\circ}$, the masses $A_{1,2}$ of the two products in coincidence were determined with a resolution better than $3 \mathrm{amu}$ (FWHM) and energy resolution of $5 \mathrm{MeV}$. In this procedure energy losses of the fragments in different passive absorbers (the target and the foils of the CORSET start detectors) is accounted for. 
The prompt $\gamma$-rays were detected with ORGAM [42], an array of high-resolution Ge detectors, individually surrounded by BGO anti-Compton shields, and PARIS [43], an array of high-efficiency $\mathrm{LaBr} 3(\mathrm{Ce})-$ $\mathrm{NaI}(\mathrm{Tl})$ phoswiches. For this experiment 10 ORGAM detector units were used, all placed at backward angles at $18 \mathrm{~cm}$ from the target center, and 10 PARIS units, 9 of them closely packed together at $38 \mathrm{~cm}$ from the target center, plus a single unit placed at $30 \mathrm{~cm}$ from the target center. All PARIS phoswiches were mounted at forward angles. A schematic drawing and a photo of the experimental setup are shown in Fig. 1.
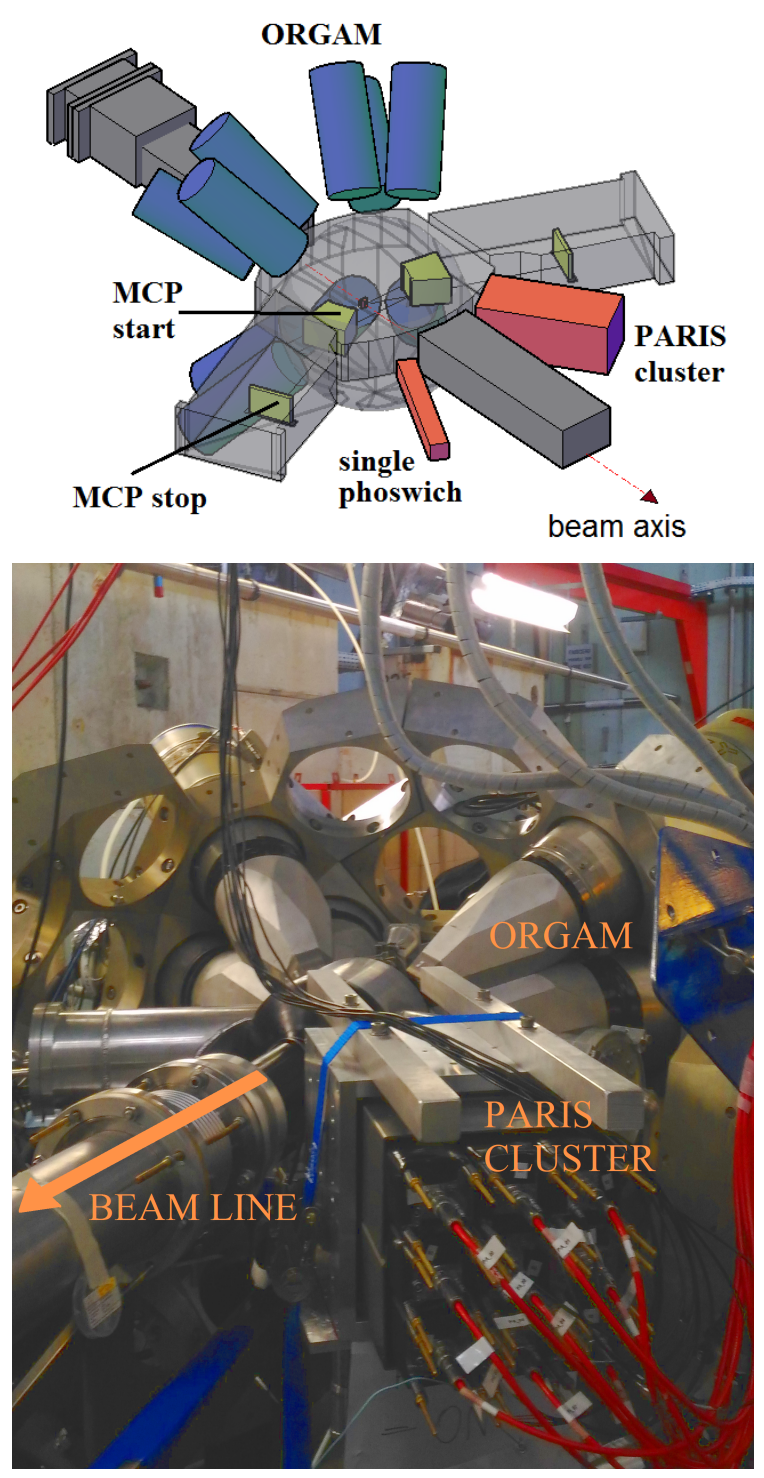

FIG. 1. A schematic drawing and a photo of the experimental setup used in the experiment. Reprinted figure from Kozulin et al. [36] with kind permission of The European Physical Journal (EPJ).)

The energy calibration of each $\gamma$-ray detector was performed using several standard radioactive sources $\left({ }^{60} \mathrm{Co}\right.$, ${ }^{137} \mathrm{Cs},{ }^{152} \mathrm{Eu}$ and $\left.{ }^{241} \mathrm{Am}-{ }^{9} \mathrm{Be}\right)$. It should be noted that the last calibration point used in the energy calibration of the PARIS detectors was at $E_{\gamma}=4.4 \mathrm{MeV}$. Therefore, the high energy calibration of PARIS was extrapolated from the low energy one. The measured photopeak efficiencies in the low energy range were $1.5 \%$ and $0.7 \%$ for ORGAM and PARIS, respectively. ORGAM has a better energy resolution ( $4 \mathrm{keV}$ at $1408 \mathrm{keV}$ ) than PARIS ( 60 $\mathrm{keV}$ at $1332 \mathrm{keV}$ ), however PARIS provides additional information by spanning a larger dynamical range (up to $20 \mathrm{MeV}$ ), compared to ORGAM (up to $2.4 \mathrm{MeV}$ ).

The readout of the PARIS detectors was accomplished with the newly designed LaBrPRO module [44]. The energy and time signals of the three setups CORSET + ORGAM + PARIS were fed into the VIPERS data acquisition system [45-49] running a VME front-end with commercial TDC and ADC modules.

Binary fragments and $\gamma$-rays were detected in singles and in prompt coincidence. The $\gamma$-ray multiplicity $M_{\gamma}$ in coincidence with $\mathrm{QE}$ and $\mathrm{FF}$ fragments were determined from the number of $\gamma$ detectors hit in the binary event (QE or FF), the so called $\gamma$-fold. The fold-to- $M_{\gamma}$ conversion was performed as it will be shown in the next sections.

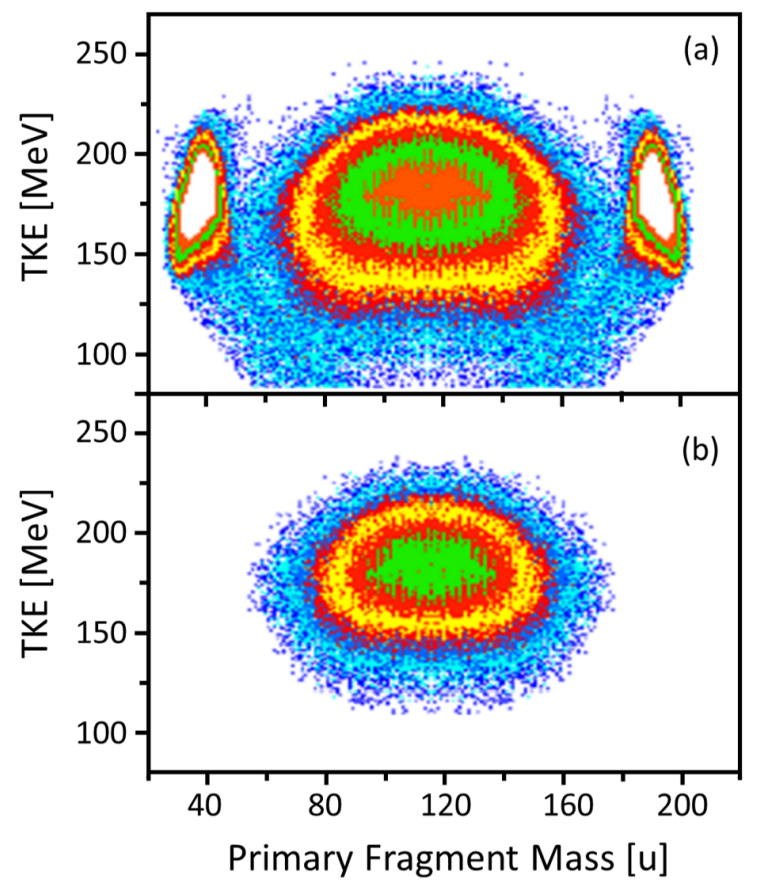

FIG. 2. (a) Mass-TKE matrix of the binary events from the reaction ${ }^{32} \mathrm{~S}\left(\mathrm{E}_{l a b}=166 \mathrm{MeV}\right)+{ }^{197} \mathrm{Au}$ without conditions and (b) applying the full momentum transfer condition and gate on FF only. 


\section{SELECTION OF BINARY REACTION CHANNELS}

If complete fusion occurs, the reaction ${ }^{32} \mathrm{~S}\left(\mathrm{E}_{l a b}=166\right.$ $\mathrm{MeV})+{ }^{197} \mathrm{Au}$ leads to the neutron-deficient ${ }^{229} \mathrm{Am}$ compound nucleus, excited at $E_{C N}^{*} \approx 43 \mathrm{MeV}$. The ultimate goal of the experiment is to study the trend of $M_{\gamma}$ when $\gamma$-ray events are gated on FF and QE events separately. In this study we widely profit of the measurements of mass and TKE of the coincident fragments and the fact that FF and QE events are well separated in the MassTKE correlation matrix.

Figure 2(a) shows the binary fragment Mass-TKE matrix obtained in this experiment for center-of-mass angles at $(90 \pm 10)^{\circ}$. The Mass-TKE matrix for binary events is reconstructed from CORSET coincidence data alone. The measurements of the event-by-event time-of-flight and the flight path of each fragment in binary coincidence are used to reconstruct the event-by-event velocity vectors $\overrightarrow{v_{1}}, \overrightarrow{v_{2}}$ of the two emerging fragments; then, from the two velocity vectors, by considering mass and momentum conservation laws, masses and energies of each couple of fragments are reconstructed. Velocity vectors are needed to obtain the mass distribution as well as to correct the $\gamma$ energy spectra for Doppler effect (see later).

By considering only the full momentum transfer (FMT) and the $\left|\overrightarrow{v_{2}}\right|$ vs. $\left|\overrightarrow{v_{1}}\right|$ event matrix it is possible to isolate the FF reaction products. The FMT selection is obtained by requiring that the sum of velocity vector projections on the reaction plane is equal to the center of mass velocity (see ref. [50] for more details). Thus, the Mass-TKE distribution in Figure 2(b), obtained requiring the FMT and selection of FF events in the velocity matrix $\left|\overrightarrow{v_{2}}\right|$ vs. $\left|\overrightarrow{v_{1}}\right|$, can be considered as originated in FF reactions being QF component negligible in this reaction. The two loci on the left and right of the FF region (Figure 2(a)) can be ascribed to the QE events.

\section{SELECTION OF $\gamma$-RAYS IN COINCIDENCE WITH BINARY REACTION CHANNELS}

By gating on specific regions of the Mass-TKE matrix, one is able to select only $\gamma$-rays associated to each reaction mechanisms, FF or QE. To extract $\gamma$ energy spectra, however, $\gamma$-ray events have to be properly processed. For instance, for both PARIS and ORGAM, gates have been considered on time signals to isolate the prompt $\gamma$-rays component of the energy spectra. The anti-coincidence condition between Ge and BGO detectors suppresses the Compton component of the ORGAM energy spectrum. An example of such spectra resulting from this procedure are shown in Figure 3. Both spectra correspond to a single ORGAM detector. In Figure 3(a) $\gamma$-rays are in coincidence with QE events; in Figure 3(b) $\gamma$-rays are in coincidence with FF events. The most visible peaks of the QE $\gamma$ spectrum can be assigned to the low energy and low angular momentum ${ }^{197} \mathrm{Au}$ transitions (marked

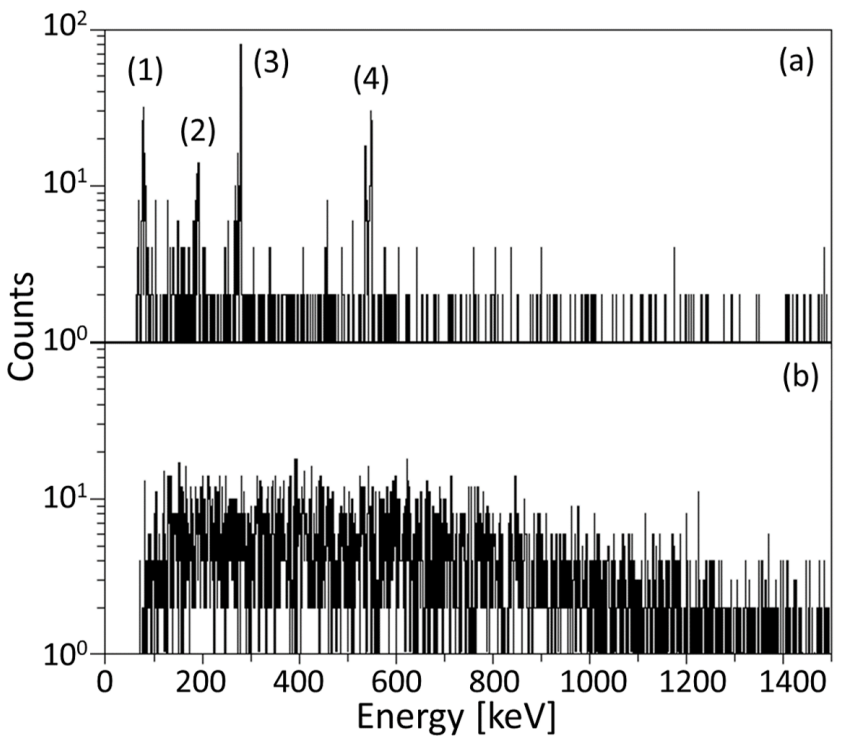

FIG. 3. (a) $\gamma$-ray energy spectra detected in coincidence with $\mathrm{QE}$ events. Marked peaks are ${ }^{197} \mathrm{Au}$ transitions. Energies of the peaks are, in order from (1) to (4): $77 \pm 2 \mathrm{keV}, 192$ $\pm 3 \mathrm{keV}, 280 \pm 2 \mathrm{keV}, 549 \pm 4 \mathrm{keV}$. Candidates ${ }^{197} \mathrm{Au} \gamma$ transitions are: $77.351 \mathrm{keV}, 191.437 \mathrm{keV}, 279.01 \mathrm{keV}, 547.5$ $\mathrm{keV}$. (b) $\gamma$-ray energy spectra detected in coincidence with FF events.

with numbers from 1 to 4) and are strongly suppressed in the spectrum in coincidence with FF events.

By taking advantage of the unambiguously assignment of QE and FF events in the Mass-TKE matrix it is possible the extraction of the QE and FF $\gamma$-fold distribution, namely, the distribution of the number of $\gamma$-rays detected per event, by gating on identified events in the Mass-TKE distribution only. In addition to the previous processing of $\gamma$-rays, the fold extracted from PARIS detectors had to be corrected for cross-talk among the phoswiches packed in a $3 \times 3$ cluster configuration. The correction has been done using a simple algorithm: if two or more neighboring detectors produce signals in a single event, their total contribution to the fold is considered to be 1 . Background has been also subtracted. An estimate of background gives $1.5 \%$ for ORGAM and $0.5 \%$ for PARIS.

\section{THE $\gamma$-RAYS FOLD DISTRIBUTIONS}

Figure 4 shows the comparison between the $\gamma$-fold distributions obtained with PARIS alone (triangles, green full line), ORGAM alone (squares, red full line) and PARIS+ORGAM considered as a single array (labeled as $\mathrm{P}+\mathrm{O}$, circles, blue full line) in coincidence with all binary events. For each distribution the yields $\mathrm{Y}_{\mathrm{i}}$ are normalized to the yield of binary events without $\gamma$-rays in coincidence (fold $=0$ ). The index $i$ denotes the PARIS, ORGAM and PARIS+ORGAM arrays. At this first step, 


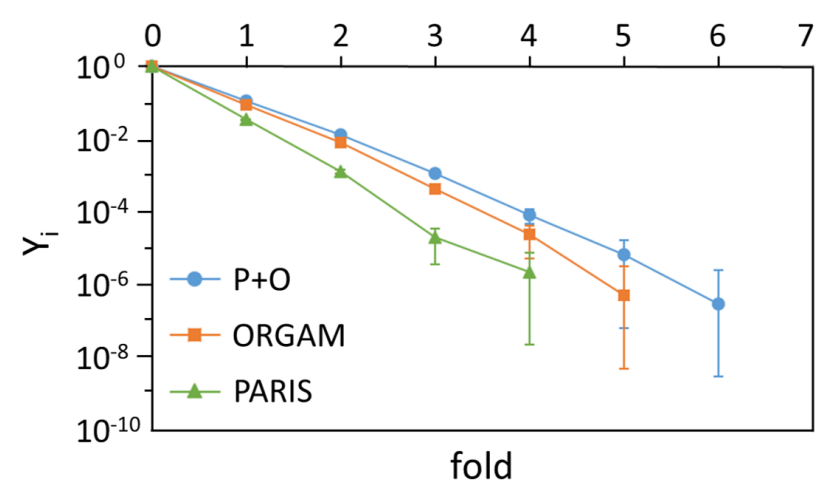

FIG. 4. $\gamma$-fold distributions for $\gamma$-rays obtained with PARIS alone (triangles, green full line), ORGAM alone (squares, red full line), and PARIS and ORGAM considered as a single array (labeled as $\mathrm{P}+\mathrm{O}$, circles blue full line) in coincidence with all binary events. Lines are drawn to guide the eyes. Counts are normalized to the total number of binary coincidence events. The average folds are: $0.12 \pm 0.04(\mathrm{P}+\mathrm{O})$, $0.03 \pm 0.02($ PARIS) and $0.09 \pm 0.03($ ORGAM $)$.

no efficiency correction is performed on these data (see later).

Average fold values are: $0.03 \pm 0.02$ for PARIS alone, $0.09 \pm 0.03$ for ORGAM alone and $0.12 \pm 0.04$ for PARIS+ORGAM $(\mathrm{P}+\mathrm{O})$. These average values are consistent with the different efficiency between PARIS and ORGAM. Even though the intrinsic efficiency of a single PARIS unit is larger than the one of an ORGAM unit, due to the different geometrical configuration, the total efficiency of the 10 used ORGAM units is larger than the total efficiency of the 10 PARIS units used. The lower average fold of the PARIS array is the straightforward consequence of the smaller total efficiency.

To verify that the differences between the three distributions are a direct consequence of the different efficiencies, the fold distributions have been normalized to the efficiency ratios. The energy averaged efficiencies are $\varepsilon_{P}=0.6 \%, \varepsilon_{O}=1.3 \% \varepsilon_{P+O}=\varepsilon_{P}+\varepsilon_{O}=1.9 \%$, for PARIS, ORGAM and PARIS+ORGAM, respectively. The PARIS and ORGAM distributions have been normalized to the $\mathrm{P}+\mathrm{O}$ one. The normalization factors depend on the efficiency ratios and fold according to the ansatz $\left(\varepsilon_{P+O} / \varepsilon_{i}\right)^{\text {fold }}$, where the index $i$ denotes the PARIS and ORGAM arrays. The new distributions are shown in Figure 5. The average fold values are now $0.13 \pm 0.06$ for PARIS, $0.13 \pm 0.04$ for ORGAM and $0.12 \pm 0.04$ for $\mathrm{P}+\mathrm{O}$. The excellent agreement between the average fold values and the shapes of the three fold distributions confirms that the differences in the raw fold distributions are due to the differences in the total efficiency. On the basis of the results above, in the following the data analysis will refer only to the PARIS+ORGAM fold distribution.

Finally, the fold distributions of $\gamma$-rays detected in coincidence with FF and QE products were extracted and are compared in Figure 6. The QE $\gamma$-fold distribution

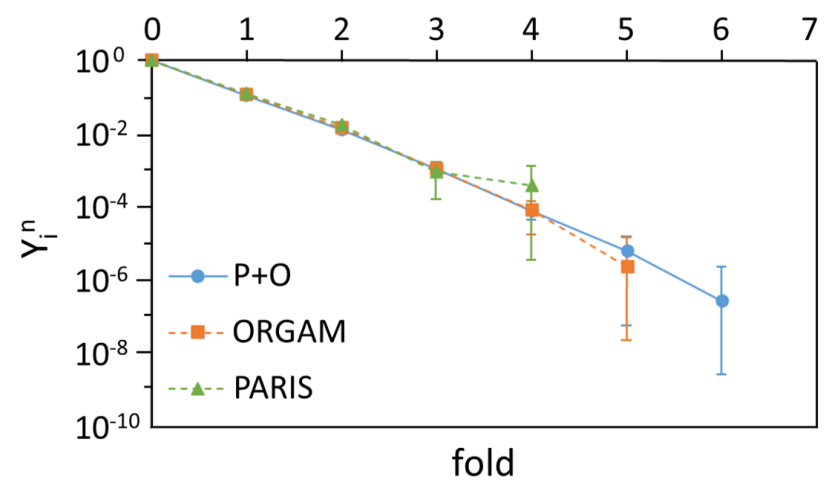

FIG. 5. $\gamma$-fold distributions for $\gamma$-rays detected in coincidence with binary events by ORGAM and PARIS together $(\mathrm{P}+\mathrm{O}$, circles, blue solid line) compared to PARIS and ORGAM distributions, renormalized for total efficiency (ORGAM, squares, red dashed line, and PARIS, triangles, green dashed line). The average folds are: $0.12 \pm 0.04(\mathrm{P}+\mathrm{O}), 0.13 \pm 0.06$ (PARIS) and $0.13 \pm 0.04$ (ORGAM).

drops much faster than the FF one, by orders of magnitude. Furthermore, in FF events, up to $6 \gamma$-rays have been detected in coincidence, whereas in QE events the maximum fold is 2 .

If we now assume that the $\gamma$-ray energy-averaged detection efficiency does not change sensibly with the binary channel (QE or FF) (the $\gamma$-rays energies span the same energy range in QE and FF, see Figure 3), these data indicate that the $\gamma$-fold distribution is an observable sensitive to the reaction time scale. The expected lower amount of orbital angular momentum transferred during the faster process explains the smaller average fold in the QE channel.

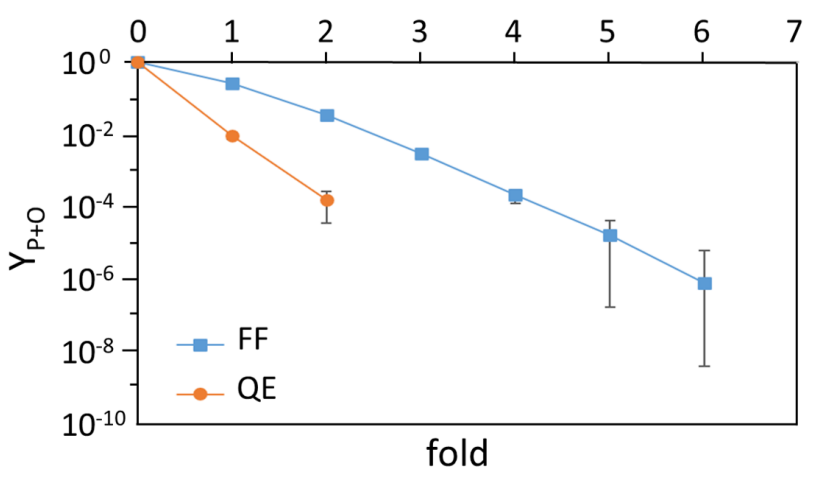

FIG. 6. $\gamma$-fold distributions detected with PARIS+ORGAM $(\mathrm{P}+\mathrm{O})$ in coincidence with $\mathrm{QE}$ and FF products. The average folds are: $0.26 \pm 0.07(\mathrm{FF}), 0.009 \pm 0.004(\mathrm{QE})$.

\section{THE $\gamma$-RAYS FOLD DISTRIBUTION AND THE RESPONSE MATRIX OF ORGAM + PARIS}

The conversion of the fold distribution into $\gamma$ multiplicity distribution is usually a complex task, which 
requires a well-constrained guess. The complexity is due to the fact that the detected number of $\gamma$ 's in each event is less than the number of $\gamma$ 's truly emitted in an event because of the limited (intrinsic and geometrical) efficiency of the $\gamma$ detectors. For instance, fold $=1$ events means that only one $\gamma$-ray is detected out of the many (unknown number) emitted. Therefore, fold $=1$ events are the overlap of a distribution of events associated with the emission of an unknown number of $\gamma$-rays, being the process of detection of stochastic origin. In other words, it is necessary to calculate what is the probability that fold $=1$, for instance, is due to a multiplicity of $1,2,3 \ldots ., n \gamma$ rays emitted. This probability represents the so called response function of the detection system. In general, we expect that with the increasing of the number of $\gamma$ 's truly emitted in the decay process also the probability of detecting more than one $\gamma$-ray increases. However, the rate of such increment is strongly dependent on the detector efficiency. Finally, to obtain an estimate of the multiplicity distribution, since we measure the fold distribution, the response function should be inverted. We will indeed proceed differently as it will be shown later.

To compute this response function in a way to include the features of PARIS+ORGAM setup, namely the geometrical efficiency and the dependence of the intrinsic efficiency on the energy of the $\gamma$-rays as measured, we prepared a Monte Carlo simulation code (SiMCa). This code computes the conditional probability $P\left(F, \mathrm{M}_{\gamma}\right)$ that a number $F$ of $\gamma$-rays, out of $\mathrm{M}_{\gamma}$ emitted in a physical event, are detected. In other words, the code calculates the probability that a fold $F$ comes from a multiplicity $\mathrm{M}_{\gamma}$.

The code starts by generating $\mathrm{M}_{\gamma} \gamma$-rays per event, from a user-defined distribution limited in the range $\mathrm{M}_{\gamma, \min }$ to $\mathrm{M}_{\gamma, \max }$, each with a randomly generated direction and energy. Being the detection probability dependent on the energy and spatial distribution of the $\gamma$-rays, the code requires in input a user-defined function for the energy distribution, while the emission direction is considered isotropic. Afterward, the code determines the amount of $\gamma$-rays that hit the detectors by taking into account the emission direction and the geometrical configuration of the detection array given by the user. For each $\gamma$-ray firing a detector, the code compares a randomly generated number in the interval $[0,1]$ with the intrinsic efficiency of the fired detector. The intrinsic efficiency in the SiMCa code follows the equation $\varepsilon(E)=A_{1} e^{\left(-E / E_{1}\right)}+\varepsilon_{1}$, in which $A_{1}, E_{1}$ and $\varepsilon_{1}$ are parameters obtained from the fit to experimental data on efficiency. Only if the random number is lower than the efficiency, the $\gamma$-ray is considered as detected. By the computed number $F$ of $\gamma$-rays detected out of $\mathrm{M}_{\gamma}$ rays generated, and by repeating the process enough times to have a sufficient precision, the probability matrix $P(F$, $\mathrm{M}_{\gamma}$ ) is generated.

To test the validity of this method, a comparison has been made with the results of the formula proposed in ref. [51] by A. Maj et al. for the probability $P\left(F, \mathrm{M}_{\gamma}\right)$.

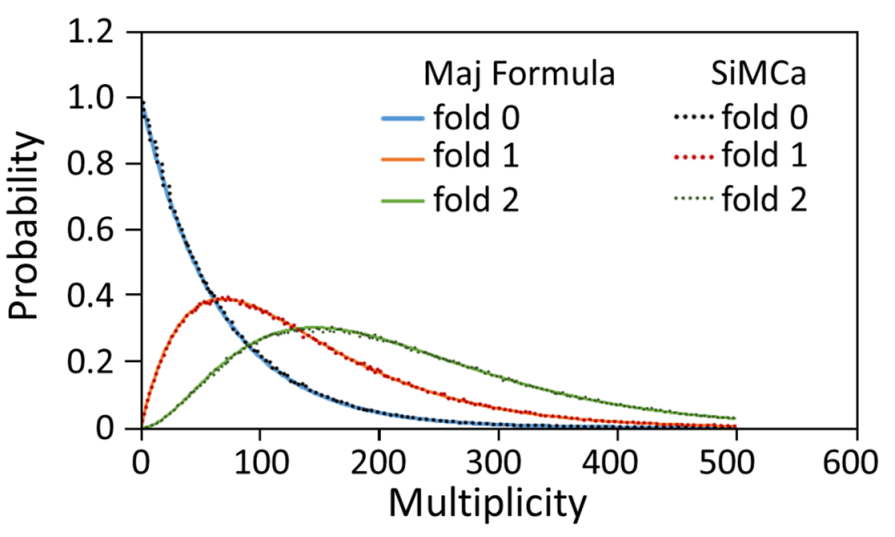

FIG. 7. Comparison between the multiplicity distribution computed with the Monte Carlo code (SiMCa, dots) and the Maj formula [51] for 3 different fold values. Only geometrical efficiency is considered.

This formula is based on a recursive algorithm and takes into account a total efficiency independent from the $\gamma$ ray energy.

A first comparison has been carried out by using in SiMCa code and in the Maj formula an intrinsic efficiency equal to 1 , independent from the $\gamma$-ray energy, i.e. the fold has been obtained by considering only the geometrical efficiency. Figure 7 shows the probabilities to have a fold $=0,1$ and 2 as function of the $\mathrm{M}_{\gamma}$ ranging from 0 to 500 . As expected, the probability to detect more than one $\gamma$-ray increases as the multiplicity increases and the maximum of the curve for each fold moves toward larger multiplicities. The distributions obtained by the two methods are indistinguishable.

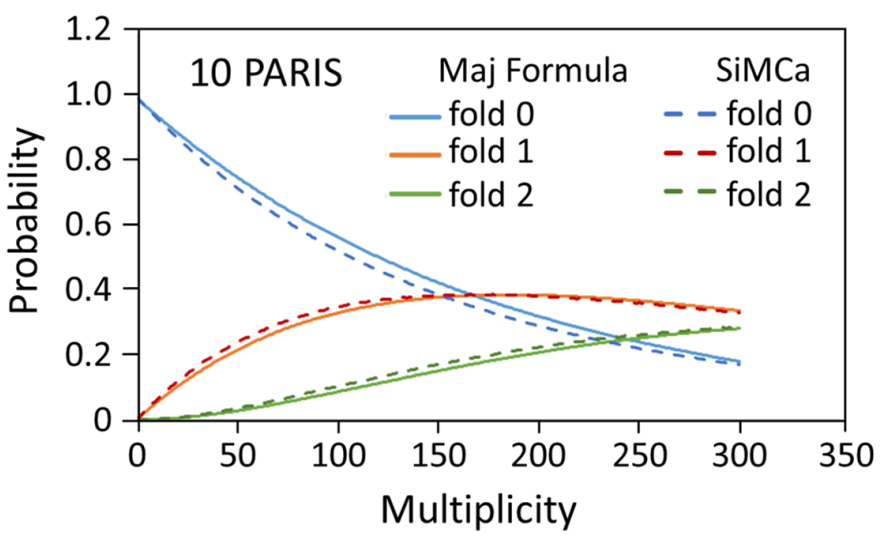

FIG. 8. Comparison between the calculation of the probability $P\left(F, \mathrm{M}_{\gamma}\right)$ made with SiMCa code, which now includes the dependence of the detection efficiency on the $\gamma$-ray energy and the formula in ref. [51] for 3 different values of fold. Dashed lines are the predictions of the SiMCa code, solid line are the results of the Maj formula.

To check the effects of the $\gamma$-ray energy dependence of the efficiency, another comparison has been made between the two methods. In the Maj formula [51] only an 
average energy-independent efficiency is accounted for. For this case, we used the efficiency obtained as the average over the energy and over different detectors. In our code we take advantage of the intrinsic flexibility of a Monte Carlo approach and we used the measured efficiency curve for each of the detectors. This flexibility is for us very valuable because ORGAM and PARIS detectors are characterized by very different intrinsic efficiencies. This means that in a single tool we can include detectors with different performances. In order to reduce the differences with respect to the Maj's formula results, in our simulations the energy spectrum is chosen as a flat distribution between 150 and $3000 \mathrm{keV}$. Figure 8 shows the comparison between the two different methods for 3 different values of the fold $=0,1$, and 2 . There are only slight differences between the two methods, which seem to disappear for increasing fold values above 2 . This is indeed a consequence of the limited angular coverage of PARIS +ORGAM. For a larger coverage of the solid angle, the differences are more marked. This behaviour can be evidenced when the number of PARIS detectors is doubled or quadrupled as shown in Figure 9(a) and (b), respectively. The curves for each fold have the same shape, but they are shifted by a relatively large amount. This means that the conversion fold-to- $\mathrm{M}_{\gamma}$ will be affected. Another remarkable feature is that the curves for fold $>0$ are narrower and the maxima progressively shift toward lower values of the multiplicity. Consequently, larger total efficiency translates in a smaller error in the conversion fold-to- $\mathrm{M}_{\gamma}$.

Even if all the comparison discussed so far have been performed using uniform distributions of $\mathrm{M}_{\gamma}$, SiMCa allows to use any kind of multiplicity distribution function. Essentially, we have developed a code able to simulate the fold distribution for a given multiplicity distribution by taking into account the geometrical and the intrinsic efficiency of all the elements constituting the detection setup. However, we need to invert the process, namely, to find the $M_{\gamma}$ distribution for a given or measured $\gamma$ fold distribution. This inversion is not straightforward and can be source of errors. Therefore, it is more convenient to assume a realistic multiplicity distribution and compare the resulting fold distribution (filtered via the response function) with the measured one. For these reasons, a search for a multiplicity distribution, grounded on some physical information, was carried out.

\section{FROM $\gamma$-RAY FOLD DISTRIBUTION TO MULTIPLICITY DISTRIBUTION}

In order to define the best guess for the true multiplicity distribution we started from the well-known method proposed by Ockels [52]. This method provides a rapidly converging algorithm to compute the first few moments of the multiplicity distribution for a given measured fold distribution and efficiency of the $\gamma$-ray detecting array. The method is particularly suited in cases where a low
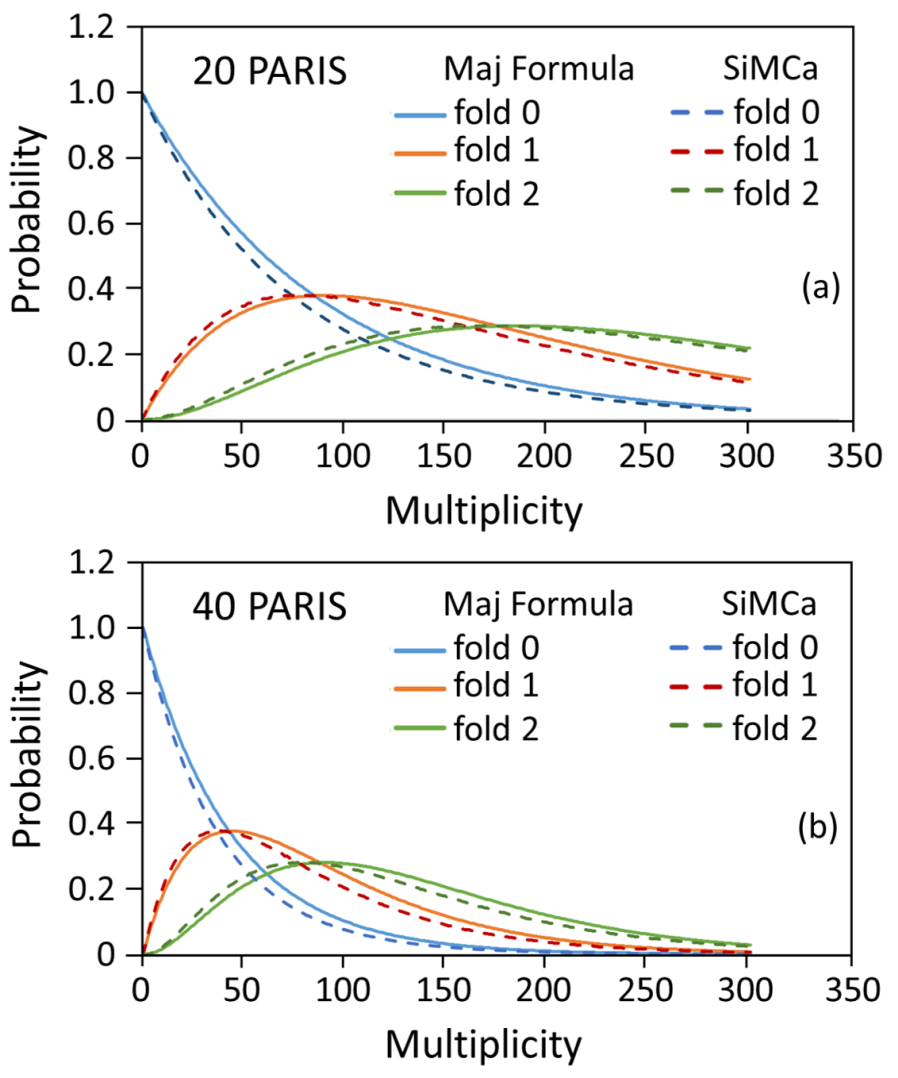

FIG. 9. Same as in Figure 8 but with a doubled (a) and quadrupled (b) PARIS efficiency.

fold $(\leq 4)$ is available. In general, the largest is the fold measured, the larger is the number of moments that can be computed. The method also provides a mean to compute errors on these shape parameters. Consistently, the error becomes bigger by increasing the order of the moment. By applying this method (equation 16 in ref. [52]) to the FF and QE fold distributions in Figure 6, we can determine some moments of the corresponding multiplicity distributions. Afterward, by fixing the moments, we can choose among several trial distributions, with moments fixed as computed, the one that reproduces at the best, once being filtered by the response function, the measured fold distributions. With this ansatz we obtain the experimental multiplicity distributions.

By using eq.16 in ref. [52], we were able to compute the first 3 moments of the experimental multiplicity distributions of the FF and QE channels. The values are shown in the Table I.

The relationships of the central moments $m_{i}$ with the shape parameters are the following:

$$
<M>=m_{1}, \sigma_{M}=\sqrt{m_{2}}, \tilde{m}_{3}=\frac{m_{3}}{m_{2}^{3 / 2}}
$$

where $\left\langle M>, \sigma_{M}\right.$ and $\tilde{m}_{3}$ are the estimate of the average, standard deviation and skewness of a candidate multiplicity distribution, respectively. The limited statistics 
TABLE I. The estimate of the first 3 central moments $m_{i}$ of the FF and QE multiplicity distributions extracted from the measured fold distributions according to Ockels [52]. $p$ and $r$ are the two parameters of a negative binomial distribution. $\mu_{3}$ is the third moment computed using eq. 3.(See text for more details)

\begin{tabular}{ccccccc}
\hline & $m_{1}$ & $m_{2}$ & $m_{3}$ & $p$ & $r$ & $\mu_{3}$ \\
\hline \hline FF & $12.5 \pm 0.4$ & $23 \pm 6$ & $100 \pm 240$ & 0.46 & 14.4 & 0.57 \\
\hline QE & $0.46 \pm 0.05$ & $1.0 \pm 0.5$ & $1.2 \pm 0.6$ & 0.54 & 0.39 & 3.34 \\
\hline
\end{tabular}

obtained in the experiment does not allow to determine the skewness and the kurtosis with good precision. For the QE channel, being the maximum measured fold $=2$, only up to three moments could be extracted [52].

\section{A. From $\gamma$-ray fold distribution to $M_{\gamma}$ distribution in the FF and QF channels}

We have tested several trial distributions, with fixed moments as in Table I, the best of which resulted to be, in both the FF and QE channels, the negative binomial defined as:

$$
f(k ; r, p)=\left(\begin{array}{c}
k+r-1 \\
k
\end{array}\right)(1-p)^{r} p^{k}
$$

with shape parameters given by:

$$
\mu_{1}=\frac{p r}{1-p}, \mu_{2}=\frac{p r}{(1-p)^{2}}, \mu_{3}=\frac{1+p}{\sqrt{p r}}
$$

By considering $m_{1}$ and $m_{2}$ from Table I as estimates of the shape parameters $\mu_{1}$ and $\mu_{2}$, respectively, the distribution parameters $p$ and $q$ were calculated and are reported in Table I for QE and FF channels. Figure 10 shows the plot of the negative binomial distributions with parameters given in Table I. Min and Max in the legend correspond to the limiting distributions due to the errors in the moments. Figure 11 shows the obtained $\gamma$-fold distributions overlapped to the measured fold distributions.

We notice a very good agreement between the experimental and computed fold distributions in both channels. However, we have to remark that the negative binomial distribution has only two parameters, $p$ and $r$. In other words, $\mu_{3}$ is fully determined once $p$ and $r$ are fixed. Indeed, we could calculate, by Ockels' method, also an estimate of the third moment $m_{3}$. However, $\mu_{3}$ as computed in eq. 3, once $p$ and $r$ are fixed from the estimate of $\langle M\rangle$ and $\sigma_{M}$ alone, is not consistent with the one computed by Ockels' method $\left(m_{3}\right)$. This reflects the fact that the estimate of the third moment $m_{3}$ is affected by an unreliable error given the low event statistics and the fact that Ockels' formula is limited to the case of an energy independent efficiency.
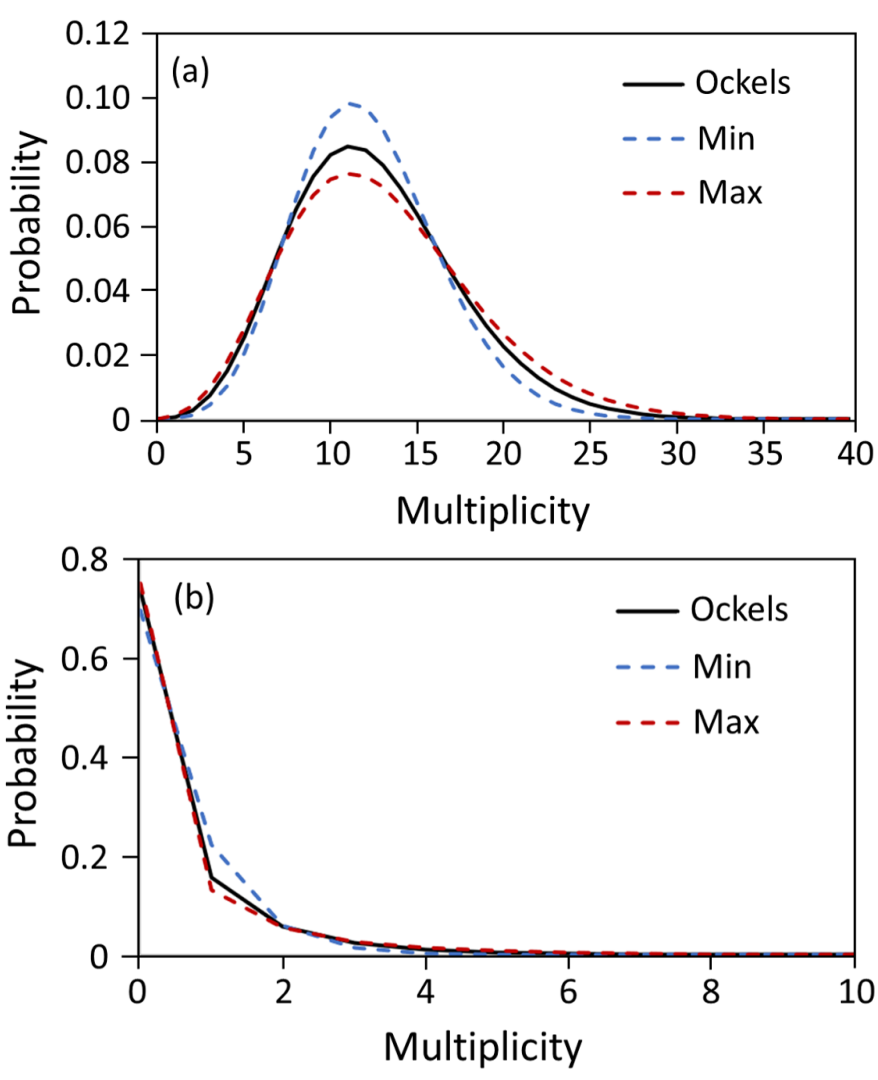

FIG. 10. Plot of the negative binomial distribution that reproduces at best the measured $\gamma$-fold distribution (a) for FF events and (b) for $\mathrm{QE}$ events. Min and Max in the legend correspond to the limiting distributions due to the errors in the shape parameters as in Table I.

B. Comparison of the extracted $M_{\gamma}$ distribution in the FF channel with the calculation of the code GEF

To gain insight on the features of the FF events producing $\gamma$-ray distributions similar to the one shown in Figure 10(a), GEF code [53] calculations have been carried out. All default GEF input parameters were kept, except for the root mean square angular momentum $l_{r m s}$ that was varied in order to better reproduce simultaneously the experimental FF mass-TKE distribution measured with CORSET and the coincident $\gamma$-ray energy spectra measured with ORGAM array. Then, the $\gamma$ ray multiplicity distribution corresponding to the GEF calculation, which better reproduces the FF channel observables, was filtered with SiMCa code, including the $\gamma$-ray response function of PARIS+ORGAM, to obtain the fold distribution. In Figure 11(a) the experimental fold distribution in the FF channel is compared with the GEF prediction and the fold obtained assuming the negative binomial distribution with parameters determined with Ockels' method shown in Figure 10(a).

In Figure 12 the distributions of FF mass (a), TKE (b) and $\gamma$-ray energy (c) are compared with experimental data. The excellent agreement with these experimental 


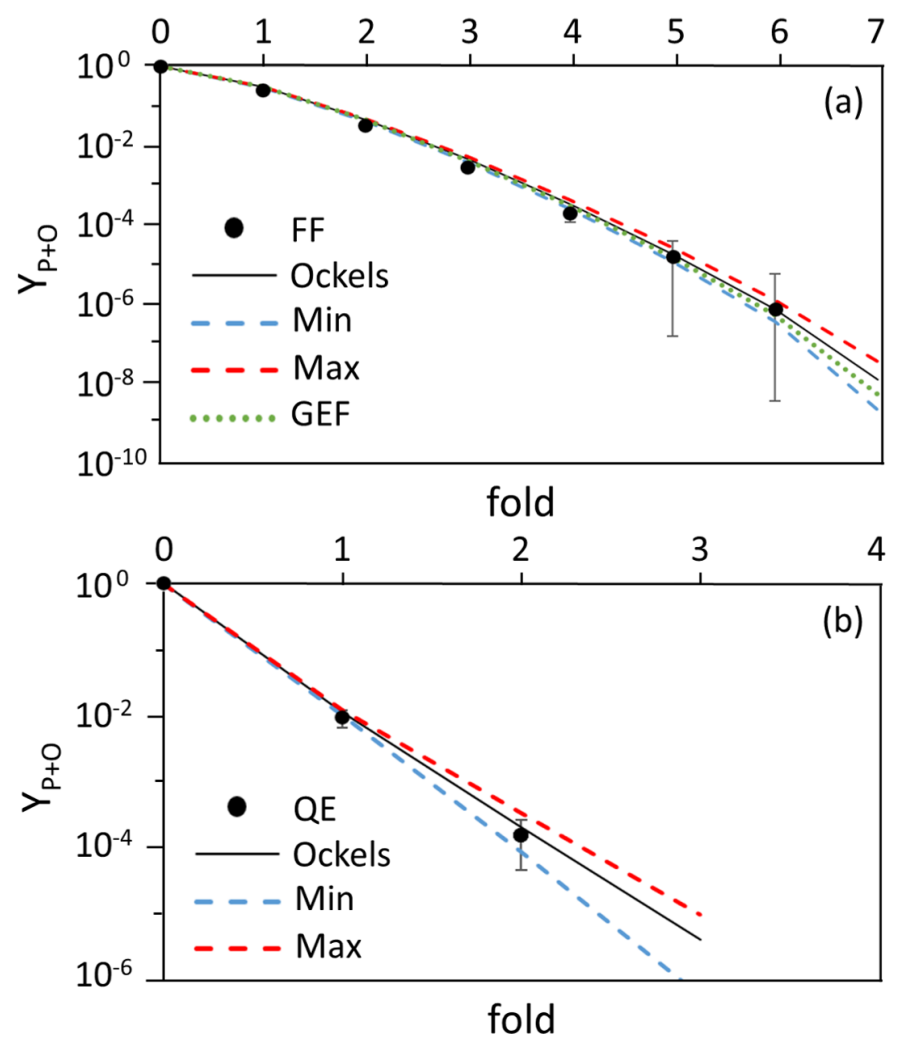

FIG. 11. $\gamma$-fold distributions in coincidence with FF (a) and QE (b) events compared to the respective fold distributions obtained after filtering the negative binomial distributions. In the case of FF events, the fold distribution obtained by the GEF code from the computed multiplicity distribution is also shown (see text). The curves labeled as Min and Max correspond to the limiting fold distributions due to the errors in the parameters as in Table I.

observables could be reached for $l_{r m s}=12.4 \hbar$. In Figure 13 the corresponding GEF $\mathrm{M}_{\gamma}$ distribution is compared to the one with the parameters from Table I. Even this comparison shows an excellent agreement. Therefore, we can conclude that the whole procedure established to extract the experimental multiplicity distribution is substantially correct, even under the condition of a limited event statistics.

\section{FROM $M_{\gamma}$ DISTRIBUTION TO ANGULAR MOMENTUM TRANSFER IN FF AND QE CHANNELS}

The final task of this data analysis is aimed at estimating, from the multiplicity distributions measured, the amount of channel orbital angular momentum $\left|\overrightarrow{l_{i}}-\overrightarrow{l_{f}}\right|$ that is transformed into the angular momentum of the final fragments in the FF and QE channels. $\overrightarrow{l_{i}}$ and $\overrightarrow{l_{f}}$ are, respectively, the entrance and exit channel orbital angular momenta and are related, by the conservation law, to the spin of the two fragments at the scission point, $\overrightarrow{J_{1}}$
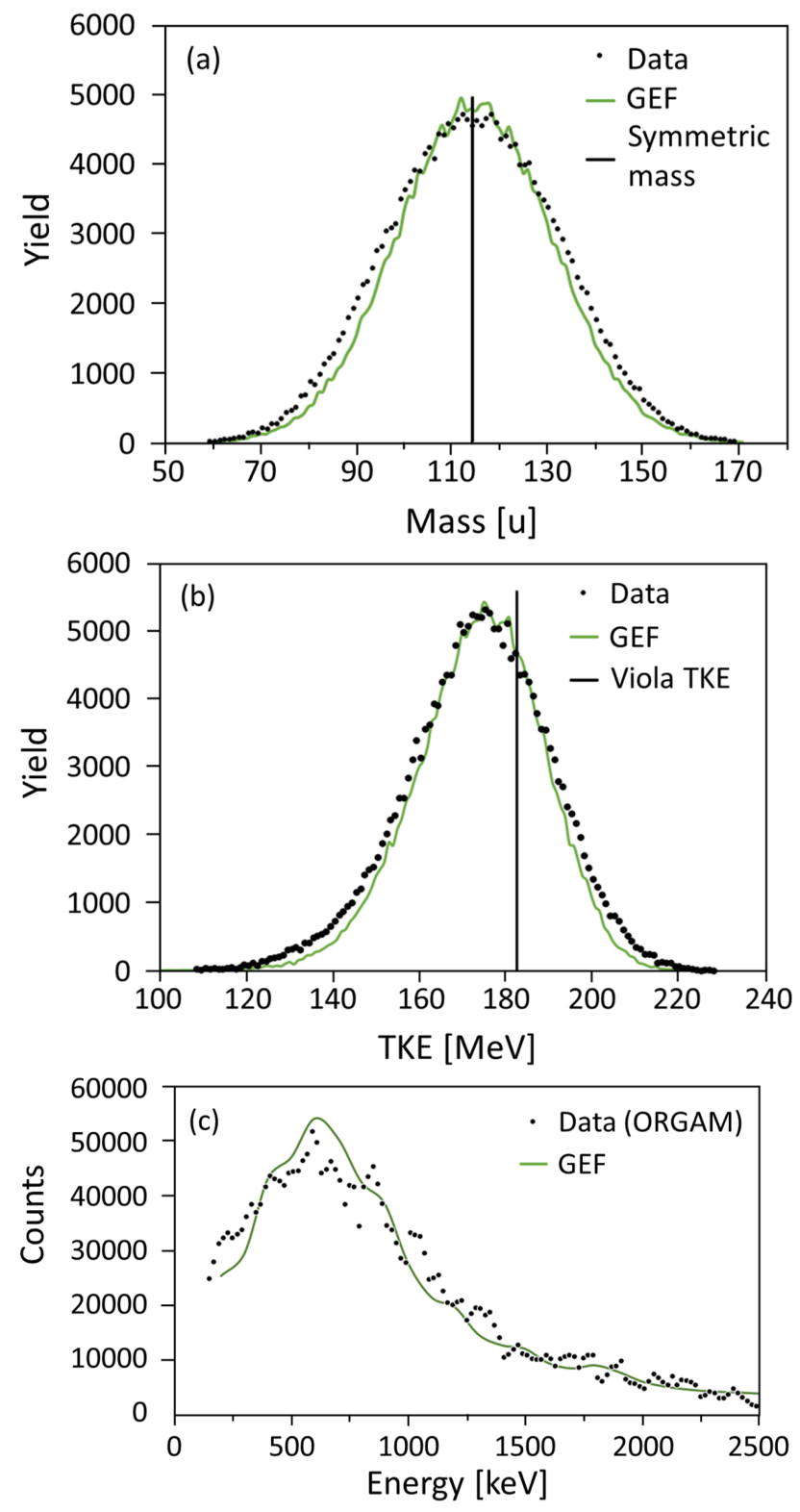

FIG. 12. Measured mass (a), TKE (b) and $\gamma$-ray energy spectra (from ORGAM only) (c) in the FF channel compared to GEF calculations for $l_{r m s}=12.4 \hbar$ and compound nucleus excitation energy $\mathrm{E}^{*}=43.5 \mathrm{MeV}$.

and $\overrightarrow{J_{2}}$, namely:

$$
\overrightarrow{l_{i}}=\overrightarrow{J_{1}}+\overrightarrow{J_{2}}+\overrightarrow{l_{f}}=\vec{J}+\overrightarrow{l_{f}}
$$

For the average values we obtain:

$$
<J>=<\left|\overrightarrow{l_{i}}-\overrightarrow{l_{f}}\right|>
$$

In other words, $\langle J\rangle$ is the average angular momentum transferred from the orbital angular momentum to the intrinsic spin of the fragments, the observable we are interested in this work. $M_{\gamma}$ distributions include contributions from both fragments but is independent of the 


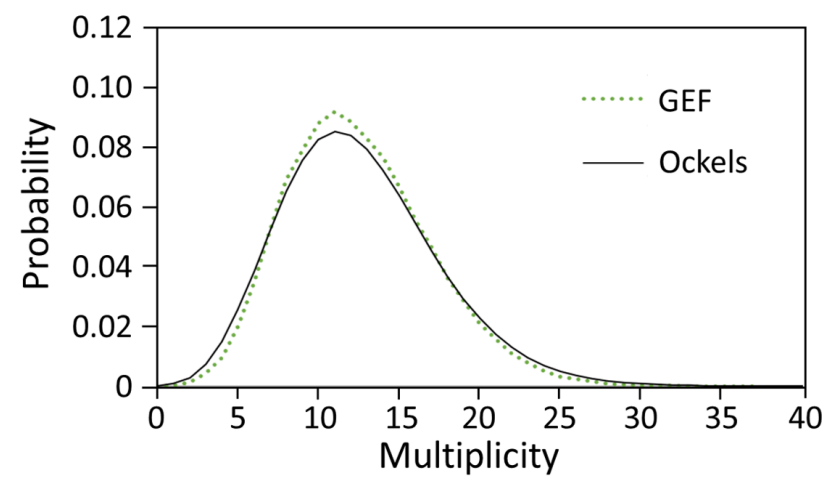

FIG. 13. Experimental $\mathrm{M}_{\gamma}$ distribution (Ockels' method) compared to the one obtained by using GEF with parameters fixed to reproduce the other measured observables (see text).

relative orientation of their respective angular momenta $\overrightarrow{J_{1}}$ and $\overrightarrow{J_{2}}$. Therefore, $\left\langle M_{\gamma}\right\rangle$ depends on the average angular momentum $\langle J\rangle$ at scission point.

The conversion from $\left\langle M_{\gamma}\right\rangle$ to $\langle J\rangle$ involves however some uncertainties. It is generally assumed that the fragments deexcite in two steps. First, emission of light particles, that carry away some angular momentum, and after $\gamma$-ray emission when the excitation energy becomes low enough to hinder particle emission. At this second stage, most of the decays proceed via stretched $E 2$ transition, corresponding to a spin change equal to $2 \hbar$, while a small number proceed via statistical $\gamma$-ray by dipole transitions which correspond to an average spin change of $0.5 \hbar$. The total amount of orbital angular momentum transferred into intrinsic angular momentum is hence given by:

$$
<J>=<\Delta I_{l p}>+<\Delta I_{\gamma}>
$$

where $\Delta I_{l p}$ and $\Delta I_{\gamma}$ are the average angular momentum carried away by all light particles and all $\gamma$-rays, respectively. Therefore, the measured $\left\langle M_{\gamma}\right\rangle$ is a function of $\langle J\rangle$ but also of the excitation energy at scission point.

Because of eq. 6, the conversion $\left\langle M_{\gamma}\right\rangle$ to $\langle J\rangle$ suffers from the lack of knowledge of the spin removed by the evaporated particles and the average multipolarities of the $\gamma$-ray transitions. In the FF channel, at the low energy of our reaction, mostly neutrons are evaporated. From the data analysis performed in [36], an average of 6 neutrons are emitted per fission decay, in agreement with GEF predictions, and can carry away on average $0.5 \hbar$. Therefore, $\Delta I_{l p}=<N_{n}><l_{n}>\simeq 3 \hbar$, where $<N_{n}>$ is the average number of evaporated neutrons and $\left\langle l_{n}\right\rangle$ is the average angular momentum carried away by a single neutron.

Accordingly, for the $\gamma$-rays we can take advantage of a common well-known expression [16, 54]:

$$
\Delta I_{\gamma}=2\left(<M_{\gamma}>-2 \alpha\right)
$$

where $\alpha$ is the average number of statistical (dipole) transitions. In this picture, stretched $E 2$ transitions take
TABLE II. Estimate of the orbital angular momentum transferred $\langle J\rangle$ according to eq. 6 in the QE and FF channels. $\left\langle M_{\gamma}\right\rangle$ is the experimental average multiplicity, $\alpha,\left\langle l_{n}\right\rangle$ and $\left\langle N_{n}\right\rangle$ are the the average number of statistical $\gamma$-ray (dipole) transitions, the average angular momentum carried away by a single neutron, the average number of evaporated

\begin{tabular}{|c|c|c|c|c|c|c|c|}
\hline & $<M_{\gamma}>$ & $\alpha$ & $\left.<l_{n}\right\rangle$ & $<N_{n}$ & $\Delta I_{l p}$ & $\Delta I_{\gamma}$ & $\langle J\rangle$ \\
\hline FF & 12.5 & 3 & 0.5 & 6 & 3 & 13 & 16 \\
\hline $\mathrm{QE}$ & 0.5 & 0 & 0 & 0 & 0 & 0 & 1.5 \\
\hline
\end{tabular}
neutrons per fission events from fragments, respectively.

away most of the angular momentum. By reviewing the pertinent literature, we used the values of $\alpha=3$ as in Table II, which also shows the transferred angular momentum as computed from eq. 6 .

In the QE channel, the two nuclei barely overlap and their kinetic energies and masses are not altered appreciably $[37,38]$. Therefore, the nuclei, after a transfer of few nucleons, continue along Coulomb-like trajectories given the expected relatively low dissipation of energy and angular momentum. We do not expect the outgoing nuclei carry enough excitation energy to induce neutron evaporation. Furthermore, only the very first few levels of the outgoing fragments can be excited. Consequently, during the $\gamma$ decay we can expect that on the average a $\gamma$-ray can carry away $1.5 \hbar$ of angular momentum. Given the measured $\left\langle M_{\gamma}\right\rangle \approx 0.5$ and $\sigma_{M_{\gamma}} \approx 1$, we reasonably deduce a narrow window of transferred angular momentum below $3 \hbar$ in the QE channel.

From Table II it is quite evident a striking difference between the angular momentum transferred in the FF and QE channels that holds regardless of the assumptions underlying eqs. 6 and 7. We could suspect about this strong contrast from the average $\gamma$ multiplicities measured in the two channels the implications of which will be discussed in the next section.

As a further support to the whole procedure used to measure $M_{\gamma}$ and to connect it to the transferred angular momentum, we observe that our result is in noteworthy agreement with the systematics proposed by Ogihara et al. [55] which connects the average excitation energy of the fission fragments $\left\langle E_{f}^{*}\right\rangle$ to the average angular momentum transferred to the fission fragments $\langle J\rangle$ via the expression:

$$
<J>=0.11<E_{f}^{*}>/ M e V+7.5
$$

For $\langle J\rangle=16 \hbar$, eq. 8 gives $\left\langle E_{f}^{*}\right\rangle=77 \mathrm{MeV}$ which is the maximum of the fission fragment excitation energy distribution derived in [36].

\section{DISCUSSION}

In the present measurements the average $\gamma$-ray multiplicity $\left\langle M_{\gamma}\right\rangle$ is used to determine the amount of angular momentum $\langle J\rangle$ introduced into internal rotation of 
the fragments produced in two-body decays over the full range of impact parameters, from $\mathrm{FF}$ to $\mathrm{QE}$ reactions. It is found that in the FF channel, $\langle J\rangle$ is much larger than in the QE channel. This result is understandable on the basis of the known picture of the FF and $\mathrm{QE}$ reaction paths. Classically, the conversion of orbital angular momentum of the entrance channel $\overrightarrow{l_{i}}$ into intrinsic angular momentum of the fragments is described as the result of tangential friction. In a first step, the nuclei slide on each other and viscous forces set in a torque which puts them into rotation. For angular momenta close to the maximum, the two nuclei undergo grazing collisions. Since the two nuclei barely overlap the kinetic energies and masses are not altered appreciably, and the nuclei continue along Coulomb-like trajectories. Hence, the reaction mechanism is confined to a very narrow gap of orbital angular momentum around the grazing angle and only few nucleons are exchanged during a rather short interaction time.

For smaller impact parameters, or lower $l$ waves, the closer contact leads to stronger damping of the kinetic energy and more extensive mass transfer. In these conditions, tangential forces continue to act until the system reaches a rolling stage in which the peripheral velocities are matched. The rolling friction slows down the rotation of the nuclei until they form a rigid body (sticking condition) $[32,37]$. According to this view of the angular momentum transfer, one expects a rapid increase of the angular momentum transferred to the fragments until the rolling state is reached with increasing interaction time. It can be demonstrated [37] that

$$
J=\frac{2}{7} l_{i}
$$

For a further increase of the interaction time, $J$ will continue to increase until it reaches its maximum value at the sticking point where

$$
J=\frac{I_{1}+I_{2}}{I_{1}+I_{2}+I_{\text {rel }}} l_{i}
$$

where $I_{1}$ and $I_{2}$ are the moment of inertia of the fragments and $I_{r e l}$ is the moment of inertia of the relative motion. For smaller $l$ waves, the system may fuse if the potential has a pocket [37]. The important point to remark here is that an increase of angular momentum transfer (or energy loss) is correlated to a growing interaction time. The maximum dissipation of angular momentum and energy correspond to complete fusion. The fused system may evolve as an evaporation residues or decays into fission. In any case, the complete fusion requires interaction times longer than peripheral reactions.

It is possible to check the consistency of the data in Table II with the above picture in mind. In the QE channel, the faster process, $\langle J\rangle$ is only few units of $\hbar$, namely almost no transfer of angular momentum occurs. Since this reaction is confined around the grazing angle $l_{g r}=25 \hbar, l_{f}$ remains confined around $25 \hbar$ and $\mathrm{TKE} \approx E_{c m}$. Differently, in the FF channel (the slower process), being $l_{i}$ fully transformed into intrinsic spin, $<l_{i}>\approx<J>=16 \hbar$. In the hypothesis of a triangular distribution, $<l_{i}>=16 \hbar$ corresponds to a maximum orbital momentum $\left\langle l_{\max }\right\rangle=24 \hbar$. In other words, the whole in-going orbital angular momentum distribution is exhausted by the FF and QE channels.

A further check on the outgoing orbital angular momentum $l_{f}$ comes from the evaluation of the TKE. With the assumption of rigid rotation of the dinuclear complex at scission, the total kinetic energy of the fragments can be expressed as the sum of their Coulomb repulsion and rotational energies:

$$
T K E=\frac{Z_{1} Z_{1} e^{2}}{d}+\frac{l_{f}\left(l_{f}+1\right) \hbar^{2}}{2 \mu d^{2}}
$$

where $d$ is the separation distance of the fragments at scission and $l_{f}$ is their relative orbital angular momentum. By taking the TKE at mass symmetry and considering the final fragment with ellipsoidal shape (with their axes of symmetry along the axis joining their center) having a deformation parameter $\beta=0.6$, we obtain $l_{f} \approx 0$. This result is in agreement with the hypothesis that the whole in-going orbital angular momentum is dissipated and appears as spin of the fragments.

A further check in favor of the conclusion that higher

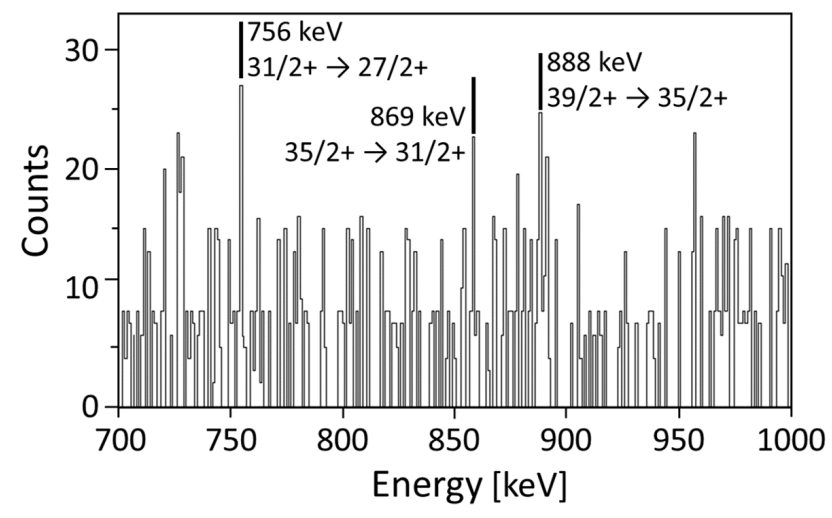

FIG. 14. High angular momentum transition $\gamma$-rays of ${ }^{129} \mathrm{La}$ in coincidence with fission fragments.

$\gamma$-ray multiplicity is related to slower interaction time and higher angular momentum transfer comes from the spectroscopy of the discrete $\gamma$ transitions in coincidence with FF and QE fragments. For this kind of analysis corrections for Doppler effect are essential. The $\gamma$-rays' energies have to be corrected for both fission fragments velocities, being impossible to know from which fragment the $\gamma$-ray originates, and the two obtained Doppler corrected spectra have been analyzed separately [56]. For instance, an entire set of $\gamma$-ray transitions have been found for the nucleus ${ }^{129} \mathrm{La}$ connecting the level with $\mathrm{E}^{*}$ $=5934.2 \mathrm{keV}$ and $\mathrm{J}^{\pi}=39 / 2^{+}$to the level with $\mathrm{E}^{*}=$ $3420.6 \mathrm{keV}$ and $\mathrm{J}^{\pi}=27 / 2^{+}$. Figure 14 shows the $\gamma$-ray energy spectra detected with the ORGAM array, in coincidence with masses in the range $(130 \pm 3 \mathrm{amu})$ and one 
low lying transition $(270 \pm 5 \mathrm{keV})$ of the ${ }^{129} \mathrm{La}$ isotope $\left(\mathrm{E}_{\gamma}=269.7 \mathrm{keV}\right)$, Doppler-corrected for the ${ }^{129} \mathrm{La}$ fragment. This result confirms the expectation that in the FF process higher angular momentum levels can be populated. We did not find the same result in the $\gamma$ spectra in coincidence with QE fragments. Consequently, also the $\gamma$-spectroscopy analysis support the idea that processes with longer time scales can convert much more orbital angular momentum into spin of the fragments. On the same foot, we can reasonably expect the same effect also in $\mathrm{QF}$ and $\mathrm{FF}$.

As a final comment we observe that, ${ }^{129} \mathrm{La}$ is a proton rich nucleus, and thus the partner must be the neutron rich nucleus ${ }^{100} \mathrm{Sr}$, whose level scheme is only fairly known. This means that looking for more proton rich nuclei (for instance the partner of the well-known ${ }^{127} \mathrm{La}$ is the unknown ${ }^{102} \mathrm{Sr}$ ) with FF or QF reaction channels, it might be possible to populate unknown neutron-rich nuclei and the reconstruction of their level scheme can be pursued by using fragment-fragment coincidences. This is a very important indication for future experiment, to be performed with larger efficiency and event statistics, because there is no other known reaction mechanism to study such nuclei.

\section{CONCLUSIONS}

$\gamma$-rays multiplicity $M_{\gamma}$ distributions were measured in the system ${ }^{32} \mathrm{~S}+{ }^{197} \mathrm{Au}$ in coincidence with the $\mathrm{QE}$ and FF fragments. The aim is to show that by selecting a faster and a slower process in the Mass-TKE matrix, the slower process is characterized by a larger transfer of the in-going orbital angular momentum. The interpretation of the data is supposed to rely on the fact that QE and FF reaction paths are known to be at the extreme of the interaction time scale: the fastest and the slowest, respectively. It is found that the average values of such $M_{\gamma}$ distributions are consistent with a full transfer of the in-going orbital angular momentum $l_{i}$ to the spin of the fragments on the FF channel, whereas only a few units of $\hbar$ are transferred to the QE fragments.

The observation that the average $\gamma$-rays multiplicity $<M_{\gamma}>$ is larger for the slower reaction channel gives support to the expectation that increasing amounts of orbital angular momentum can be transferred only by selecting slower and slower processes. It is important to remark that the observed quantities are the lower moments of the multiplicity distribution (mean and variance). These quantities cannot therefore be obtained on an event-by-event basis, but only as an average over a sample of events. Consequently, those averages cannot be used for the reverse process of data analysis, namely, the selection of a slower process by gating on the multiplicity distribution. However, the most direct observable is the $\gamma$-fold distribution. Figure 6 is thus the key result of this work and shows that gating on higher fold processes favors the selection of the slower process. This result suggests that the $\gamma$-ray probe can play a very important role in disentangling $\mathrm{FF}$ and $\mathrm{QF}$ in the regions of the mass-TKE matrix where they are overlapped. The expectation is that QF is a process faster than FF and should give rise to a lower $\gamma$ multiplicity, namely, smaller $\gamma$-fold values. To attain this separation a large efficiency for $\gamma$-ray detection would be mandatory.

As a by-product of this analysis, we have found out that neutron-rich (possibly unknown) nuclei can be populated as partners of proton-rich nuclei. This means that a completely new spectroscopy can be accessible by using fragment-fragment coincidences.

For future plans, it is crucial to test this method in condition of major interest: when FF and QF are overlapped with comparable intensity in the same mass region. However an intermediate step would be to measure the $\gamma$-fold distribution in systems where QF is dominant. It would also be important to benefit of the properties of the QF to populate unknown neutron-rich nuclei. Reactions can be chosen carefully to populate neutron-rich regions of the nuclide chart of specific interest, like the one of interest from the r-process. However, it is evident that the experimental condition must be kept at the optimum and that much larger $\gamma$-ray detection efficiency and event statistics are necessary.

\section{ACKNOWLEDGMENTS}

We would like to thank the IPNO accelerator group for the excellent beam quality and smooth running throughout the experiment. The use of detectors from the Gammapool European Spectroscopy and France UK (IN2P3/STFC) LoanPool Resources through the ORGAM (OrsayGammaArray) project is gratefully acknowledged. We also want to acknowledge the PARIS ongoing collaboration. This work was supported by Istituto Nazionale di Fisica Nucleare (INFN, Italy), FLNR, JINR, the IN2P3-JINR Agreement No14-90 and 00-50, the ENSAR2 project "N-SI-88", by the financial contribution of Romania to the Joint Institute of Nuclear Research (JINR-RO) to the 03-5-1094-2010/2016 and 03-51130-2017/2021 themes, by the Polish National Science Centre under Contract No.2013/08/M/ST2/00257 and the French LEA COPIGAL project.

\section{REFERENCES}

[1] Yu. Ts. Oganessian, A. Sobiczewski and G. M. TerAkopian, Phys. Scr. 92, 023003 (2017) and references therein. 
[2] S. Hofmann et al. , Eur. Phys. J. A 52, 180 (2016).

[3] R.C. Barber, H.W. Gaggeler, P.J. Karol, H. Nakahara, E. Vardaci, E. Vogt, Pure Appl. Chem. 81, 1331 (2009).

[4] R.C. Barber, P.J. Karol, H. Nakahara, E. Vardaci, E.W. Vogt, Pure Appl. Chem. 83, 1485 (2011)

[5] P.J. Karol, R.C. Barber, B.M. Sherril, E. Vardaci, T. Yamazaki, Pure Appl. Chem. 88, 139 (2016).

[6] P.J. Karol, R.C. Barber, B.M. Sherril, E. Vardaci, T. Yamazaki, Pure Appl. Chem. 88, 155 (2016).

[7] E. Vardaci, M. Itkis, I.M. Itkis, G. Knyazheva, E.M. Kozulin, J. Phys. G: Nucl. and Part. Phys. 46, 103022 (2019).

[8] E. Vardaci et al., Eur. Phys. J. A 43, 127 (2010).

[9] A. Di Nitto et al., Eur. Phys. J. A 47, 83 (2011).

[10] E. Vardaci, P.N. Nadtochy, A. Di Nitto, A. Brondi, G. La Rana, R. Moro, et al., Phys. Rev. C 92, 034610 (2015).

[11] E. Vardaci, A. Di Nitto, P.N. Nadtochy, G. La Rana, J. Phys. G: Nucl. Part. Phys. 46, 115111 (2019).

[12] M.G. Itkis, E. Vardaci, I.M. Itkis, G.N. Knyazheva, E.M. Kozulin, Nucl. Phys. A 944, 204 (2015).

[13] E.M. Kozulin et al., Phys. Rev. C 89, 014614 (2014).

[14] E.M. Kozulin, V.I. Zagrebaev, G.N. Knyazheva, I.M. Itkis, K.V. Novikov, M.G. Itkis, S.N. Dmitriev, I.M. Harca, A.E. Bondarchenko, A.V. Karpov, V.V. Saiko, E. Vardaci, Phys. Rev. C 96, 064621 (2017).

[15] A. Di Nitto et al., Phys. Lett. B 784, 199 (2018).

[16] R. Bock et al., Nucl. Phys. A 388, 334 (1982).

[17] J. Tõke et al., Nucl. Phys. A 440, 327 (1985).

[18] W. Q. Shen et al., Phys. Rev. C 36, 115 (1987).

[19] R. Butsch, D.J. Hofman, C.P. Montoya, P. Paul and M. Thoennessen, Phys. Rev. C 44, 1515 (1991).

[20] R. du Rietz, D.J. Hinde, M. Dasgupta, R.G. Thomas, L.R. Gasques, M. Evers, N. Lobanov and A. Wakhle, Phys. Rev. Lett. 106, 052701 (2011).

[21] G.N. Knyazheva, I.M. Itkis, E.M. Kozulin, J. of Phys.: Conf. Series 515, 012009 (2014).

[22] A. Wakhle, C. Simenel, D.J. Hinde, M. Dasgupta, M. Evers, D.H. Luong, R. du Rietz and E. Williams Rev. Lett. 113, 182502 (2014).

[23] C. Simenel, A.S. Umar, Prog. Part. Nucl. Phys. 103, 19 (2018).

[24] D. Hilscher and H. Rossner Ann. Phys. (France) 17, 471 (1992) and references therein

[25] D. Jacquet and M. Morjean Prog. Part. Nucl. Phys. 63, 155 (2009) and references therein

[26] A. Gavron et al., Phys. Rev. C 35, 579 (1987).

[27] D.J. Hinde, H. Ogata, M. Tanaka, T. Shimoda, N. Takahashi, A. Shinohara, S. Wakamatsu, K. Katori, H. Okamura, Phys. Rev. C 39, 2268 (1989).
[28] P.N. Nadtochy et al., Phys. Lett. B 685, 258 (2010).

[29] R. Moro et al., Eur. Phys. J. A 48, 159 (2012).

[30] A. Di Nitto et al., Phys. Rev. C 93, 044602 (2016).

[31] A. Di Nitto et al., Nucl. Phys. A 971, 21 (2018).

[32] V.V. Volkov, Phys. Rep. 44, 93 (1978).

[33] B.B. Back, S. Bjørnholm, T. Døssing, W.Q. Shen, K.D. Hildenbrand, A. Gobbi and S.P. Sørensen, Phys. Rev. C 41, 1495 (1990).

[34] H. Paşca, Sh.A. Kalandarov, G.G. Adamian, N.V. Antonenko, Phys. Rev. C 96, 044611 (2017).

[35] B.B. Back, R.R. Betts, J.E. Gindler, B.D. Wilkins, S. Saini, M.B. Tsang, C.K. Gelbke, W.G. Lynch, M.A. McMahan and P.A. Baisden, Phys. Rev. C 32, 195 (1985).

[36] E.M. Kozulin, I.M. Harca, E. Vardaci et al., Eur. Phys. J. A 56, 6 (2020).

[37] R. Bass, Nuclear Reactions with Heavy Ions (Berlin:Springer-Verlag) (1980)

[38] V. Zagrebaev, Heavy Ion Reactions at Low Energies, Lecture Notes in Physics, vol. 963, A. Denikin, A. Karpov, N. Rowley (eds), Springer Nature Switzerland (2019)

[39] M.G. Itkis and A.Ya Rusanov, Phys. Part. Nucl. 29, 160 (1998).

[40] V.E. Viola, K. Kwiatkowski, M. Walker, Phys. Rev. C 31, 1550 (1985).

[41] E.M. Kozulin, A.A. Bogachev, M.G. Itkis, I.M. Itkis, G.N. Knyazheva, N.A. Kondratiev, L. Krupa, I.V. Pokrovsky, E.V. Prokhorova, Instrum. Exp. Tech. 51, 44 (2008).

[42] P.J. Nolan, F.A. Beck, D.B. Fossan, Annu. Rev. Nucl. Part. Sci. 45, 561 (1994).

[43] A. Maj et al., Acta Phys. Pol. B 40565 (2009).

[44] C. Boiano, F. Camera, S. Brambilla et al., NSS, IEEE, N10-115 (2010).

[45] M. Romoli et al., IEEE Trans. Nucl. Sci. 52, 1860 (2005).

[46] C. Signorini et al., Eur. Phys. J. A 44, 63 (2010).

[47] D. Pierroutsakou et al., Eur. Phys. J. Special Topics 150, 47 (2007).

[48] M. Mazzocco et al., Eur. Phys. J. Special Topics 150, 37 (2007).

[49] N. Patronis, A. Pakou, D. Pierroutsakou, A.M. SánchezBenítez, L. Acosta, N. Alamanos et al., Phys. Rev. C 85, 024609 (2012).

[50] E. M. Kozulin et al., Phys. Rev. C 86, 044611 (2012).

[51] A. Maj et al., Nucl. Phys. A 571, 185 (1994).

[52] W.J. Ockels, Z. Phys. A 286, 181 (1978).

[53] K.-H. Schmidt, B. Jurado, C. Amouroux, and C. Schmitt, Nucl. Data Sheets 131, 107 (2016).

[54] R.P. Schmitt, G. Mouchaty and D.R. Haenni, Nucl. Phys. A 427, 614 (1984)

[55] M. Ogihara et al., Z. Phys. A 335, 203 (1990).

[56] E.M. Kozulin et al., Eur. Phys. J. A 52, 293 (2016). 